\title{
Mesenteric Panniculitis: Case Report
}

Labbi I1 ${ }^{*}$, Dkhissi $\mathrm{Y}^{1}$, ElBouhaddouti $\mathrm{H}^{1}$, Mouaqit $\mathrm{O}^{1}$, Ousadden $\mathrm{A}^{1}$, AIT Taleb $\mathrm{K}^{1}$, Benjelloun $\mathrm{E}^{1}$

${ }^{1}$ Department of general surgery A, CHU Hassan II FES, 30000, Morocco

DOI: $10.36347 /$ sasjs.2021.v07i04.003

| Received: 07.03.2021 | Accepted: 26.03.2021 | Published: 02.04.2021

*Corresponding author: Labbi I

Abstract

Case Report

Introduction: Mesenteric panniculitis is a nonspecific inflammatory process affecting the fatty tissue at the root of the mesentery. It is characterized by the association of inflammation, necrosis or fibrosis involving the adipose tissue of the bowel mesentery. The pathophysiology of this disease remains unclear. When symptomatic, patients may present with abdominal pain, palpable abdominal mass or intestinal obstruction. The disease remains asymptomatic in 30 to $50 \%$ of cases. Abdominal CT plays an important role in suggesting the diagnosis. Medical treatment may consist of therapy with anti inflammatory or immunosuppressive agents. Surgical treatment should be exclusively attempted when intestinal obstruction or ischemia occur. Mesenteric panniculitis usually has an uneventful clinical course and resolves spontaneously in a variable delay. Observation: we report the case of a 35-year-old patient with no pathologic history, admitted to the emergency departments for the management of intense epigastric abdominal pain associated with a few episodes of vomiting. The diagnosis of mesenteric panniculitis was retained on an abdominal CT, the treatment consisted of corticosteroid therapy with good evolution. Conclusion: Mesenteric panniculitis is a rare pathology. The diagnosis is most often mentioned on imaging and requires histological confirmation given of the many differential diagnoses on imaging. Only the symptomatic forms require the initiation of treatment. The natural course of PM is good.

Keywords: Mesenteric panniculitis; Retractilemesenterite; Sclerosingmesenterite.

Copyright $\odot 2021$ The Author(s): This is an open-access article distributed under the terms of the Creative Commons Attribution 4.0 International License (CC BY-NC 4.0) which permits unrestricted use, distribution, and reproduction in any medium for non-commercial use provided the original author and source are credited.

\section{INTRODUCTION}

Mesenteric panniculitis also named retractilemesenterite is a nonspecific inflammatory process affecting the fatty tissue at the root of the mesentery [1]. This term is also used to describe the clinical and imaging findings in this disorder. It is characterized by the association of inflammation, necrosis or fibrosis involving the adipose tissue of the bowel mesentery. The pathophysiology of this disease remains unclear despite associations with inflammatory diseases or malignancies, especially lymphomas that have been described. When symptomatic, patients may present with abdominal pain, palpable abdominal mass or intestinal obstruction. The disease remains asymptomatic in 30 to $50 \%$ of cases. Abdominal CT plays an important role in suggesting the diagnosis and can be useful in distinguishing the several conditions that can mimic mesenteric panniculitis [2, 3]. Nevertheless, pathologic examination of surgical excisional biopsies or sometimes percutaneous biopsies remains necessary to confirm the diagnosis and exclude an underlying infection or malignancy $[4,5]$. Medical treatment may consist of therapy with anti inflammatory or immunosuppressive agents and can be proposed in highly symptomatic diseases. Surgical treatment should be exclusively attempted when intestinal obstruction or ischemia occur. Most of the time, it consists in intestinal derivation or segmental resection because complete excision of the lesions is often not possible. Mesenteric panniculitis usually has an uneventful clinical course and resolves spontaneously in a variable delay

\section{CASE PRESENTATION}

We report the case of a 35-year-old patient with no pathologic history, admitted to the emergency departments for the management of intense epigastric abdominal pain associated with nausea and a few episodes of vomiting, she had no change in bowel habits and was passing flatusand stools.

The physical examination found a conscious patient, with tachycardia at 110 beats per minute febrile at $38^{\circ}$ with epigastric tenderness, the rest of the clinical examination is unremarkable. A Laboratory datarevealeda hyperleukocytosis at 16000 with a CRP at 120 , normal lipasemia as well as the rest of the assessment. 
Computed tomography (CT) of the abdomen was performed using reconstructed slice thickness of 5 $\mathrm{mm}$ after intravenous (iv) contrast administration, which showed a focal increase in density of the mesenteric fat with stranding in the supra-umbilical region, which was most probably inflammatory in origin and suggestive of mesenteric panniculitis(figure 1).

The patient was started on prednisone $40 \mathrm{mg}$ daily and was followed-up closely. Her symptoms gradually decreased in intensity and pain disappeared totally within 1 week.

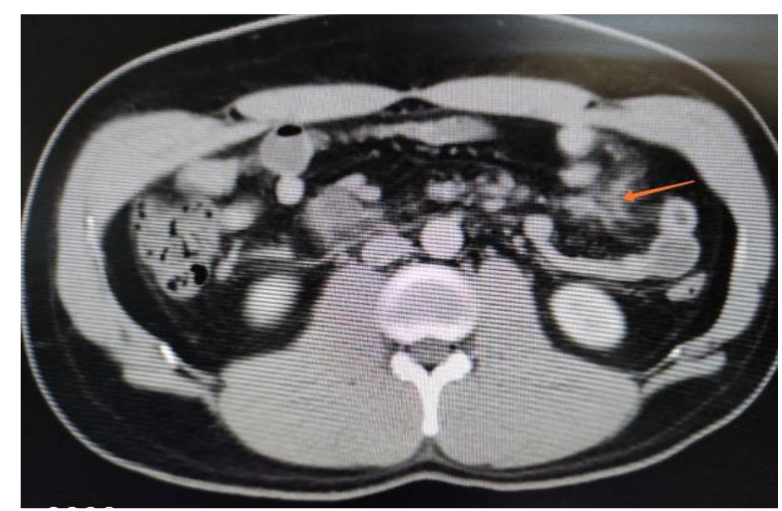

Fig-1: CT scan in the axial plane after IV contrast administration shows a focal increase in density of the mesenteric fat (arrow)

\section{DISCUSSION}

Mesenteric panniculitis is a rare inflammatory condition that is characterized by chronic and nonspecific inflammation of the adipose tissue of the intestinal mesentery, first described in the medical literature in 1924 as "retractile mesenteritis". Since that time, alternative names have been used to describe the condition including mesenteric panniculitis, retractile mesenteritis and mesenteric lipodystrophy. These names denote the predominant features of the disease process in the mesentery. The disease mainly affects people in their fifties, with, with a male-to-female ratio of $2-3: 1$.

The pathogenic mechanism of mesenteric panniculitis seems to be a nonspecific response to a wide variety of stimuli. Although various causal factors have been identified, the precise etiology remains unknown ,Furthermore, the disease is related to other factors, such as mesenteric thrombosis, mesenteric arteriopathy, drugs, thermal or chemical injuries, vasculitis, avitaminosis, autoimmune disease, retained suture material, pancreatitis, bile or urine leakage, hypersensitivity reactions, and even bacterial infection $[4,5]$ Other factors, such as gallstones, coronary disease, cirrhosis, abdominal aortic aneurysm, peptic ulcer, or chylousascitis, have also been linked to this disease[6]. More recent studies have shown a strong relationship between tobacco consumption and panniculitis [5].
Retractile mesenteritis has been associated with a number of malignant diseases such as lymphoma, lung cancer, melanoma, colon cancer, renal cell cancer, myeloma, gastric carcinoma, chronic lymphocytic leukemia, Hodgkin's disease, large cell lymphoma (giant-cell carcinoma), carcinoid tumor, and thoracic mesothelioma[4, 5, 7, 8].

In over $90 \%$ of cases, mesenteric panniculitis involves the small-bowel mesentery, although it may sometimes involve the sigmoid mesentery [9]. On rare occasions, it may involve the mesocolon, peripancreatic region, omentum, retroperitoneum or pelvis [10].

The clinical presentation of mesenteric panniculitis is highly variable. Some patients have few or no noticeable symptoms. The most common symptom is abdominal pain.The diagnosis of mesenteric panniculitis may be made incidentally following a CT scan of the abdomen, generally for the evaluation of abdominal pain.

Histologically, the disease progresses in three stages [4]. The first stage is mesenteric lipodystrophy, in which a layer of foamy macrophages replaces mesenteric fat. Acute inflammatory signs are minimal or non-existent; the disease tends to be clinically asymptomatic and prognosis is good. In the second stage, termed mesenteric panniculitis, histology reveals an infiltrate made up of plasma cells and a few polymorphonuclear leukocytes, foreign-body giant cells, and foamy macrophages. The final stage is retractile mesenteritis, which shows collagen deposition, fibrosis, and inflammation.

Mesenteric panniculitis resolves spontaneously in most cases, however, palpable masses may often be found between 2 and 11 years after diagnosis, especially in patients with associated comorbidity [8]. In such cases, several types of treatment have been proposed but no consensus has been established. In general, treatment has been reserved for symptomatic cases. Incidental masses may be observed and left untreated. Therapy is individualized on a case by case basis. Treatment may be attempted with a variety of drugs including steroids, thalidomide, cyclophosphamide, progesterone, colchicine, azathioprine, tamoxifen, antibiotics and emetine, or radiotherapy, with different degrees of success $[11,12]$. Surgery may be attempted if medical therapy fails or in the presence of life-threatening complications such as bowel obstruction or perforation [13].

\section{CONCLUSION}

Mesentericpanniculitis is a rare clinical entity that occurs independently or in association with other disorders. Diagnosis of this nonspecific, benign inflammatory disease is a challenge to gastroenterologists, radiologists, surgeons and pathologists. CT features of the disease, usually highly 
suggestive, have recently been delineated clearly. Open biopsy seems rarely necessary. There is no standardized treatment, only thesymptomatic forms require the initiation of treatment, and it may consist of antiinflammatory or immunosuppressive agents. We recommend resection only when the advanced inflammatory changes become irreversible or in cases of bowel obstruction. Overall prognosis is usually good and recurrence seems to be rare.

\section{Conflict of interest}

No conflict of interest.

\section{Source of funding}

No source of funding or sponsor.

\section{Ethical approval}

Written informed consent was obtained from the patient.

\section{Consent}

Written informed consent was obtained from the patient.

\section{Author contributions}

All authors have approved the final article

\section{REFERENCES}

1. Ogden WW, Bradburn 2nd DM, Rives JD. Pannicultis of the mesentery. Ann Surg. 1960; 151:659-68.

2. Emory TS, Monihan JM, Carr N J. SclerosingMesenteritis, Mesenteric Panniculitis and Mesenteric Lipodystrophy: A Single Entity? Am J Surg Pathol. 1997; 21:392-98.

3. Akram S, Pardi DS, Schaffner JA. Sclerosingmesenteritis: clinical features, treatment, and outcome in ninety-two patients. Clin GastroenterolHepatol. 2007; 5:589-96.

4. Delgado Plasencia L, Rodríguez Ballester L, López-Tomassetti Fernández EM, Hernández
Morales A, Carrillo Pallarés A, Hernández Siverio N. [Mesentericpanniculitis: experience in our center] Rev Esp Enferm Dig. 2007; 99:291-297.

5. Daskalogiannaki M, Voloudaki A, Prassopoulos P, Magkanas E, Stefanaki K, Apostolaki E, Gourtsoyiannis N. CT evaluation of mesenteric panniculitis: prevalence and associated diseases. AJR Am J Roentgenol. 2000; 174:427431.

6. Patel N, Saleeb SF, Teplick SK. General case of the day. Mesenteric panniculitis with extensive inflammatory involvement of the peritoneum and intraperitoneal structures. Radiographics. 1999; 19:1083-1085.

7. Cuff R, Landercasper J, Schlack S. Sclerosingmesenteritis. Surgery. 2001; 129:509510.

8. Shah AN, You CH. Mesenteric lipodystrophy presenting as an acute abdomen. South Med J. 1982; 75:1025-1026.

9. McCrystal DJ, O'Loughlin BS, Samaratunga H. Mesenteric panniculitis: a mimic of malignancy. Aust N Z J Surg. 1998;68:237-239

10. Akram S, Pardi DS, Schaffner JA, Smyrk TC. Sclerosingmesenteritis: clinical features, treatment, and outcome in ninety-two patients. Clin GastroenterolHepatol. 2007;5:589-596; quiz 523524

11. Parra-Davila E, McKenney MG, Sleeman D, Hartmann R, Rao RK, McKenney K, Compton RP. Mesenteric panniculitis: case report and literature review. Am Surg. 1998;64:768-771

12. Miyake H, Sano T, Kamiya J, Nagino M, Uesaka K, Yuasa N, Oda K, Nimura Y. Successful steroid therapy for postoperative mesenteric panniculitis. Surgery. 2003;133:118-119

13. Gu GL, Wang SL, Wei XM, Ren L, Li DC, Zou FX. Sclerosingmesenteritis as a rare cause of abdominal pain and intraabdominal mass: a cases report and review of the literature. Cases J. 2008; $1: 242$. 